\title{
Commentary
}

\section{Inflammation and Remyelination in the Central Nervous System}

\section{A Tale of Two Systems}

\author{
Francesca Ruffini, Timothy E. Kennedy, and \\ Jack P. Antel \\ From the Montreal Neurological Institute, McGill University, \\ Montreal, Quebec, Canada
}

Multiple sclerosis (MS) is a chronic disorder characterized in its initial stages by episodes of neurological dysfunction (relapses). These relapses are considered to reflect injury primarily of the central nervous system (CNS) myelin or its cell of origin the oligodendrocyte (OGC), mediated by an inflammatory response initiated by autoreactive lymphocytes recognizing neural (myelin) constituents. Formation of such inflammatory lesions can be ablated by systemic immunotherapy. Relapses are followed by variable degrees of functional recovery over subsequent days to weeks. Although the focus of this commentary will relate to the contribution of remyelination to the recovery process, this process likely reflects multiple factors including resolution of the inflammation, changes in properties of demyelinated axons, and functional reorganization of the CNS. Pathological analysis of active MS lesions documents that remyelination is occurring even in presence of concurrent inflammation. ${ }^{1}$ Thus both injury and repair are ongoing within the same inflammatory lesion raising the intriguing conclusion that the inflammatory response is both contributing to the injury and repair processes. In an estimated $50 \%$ of cases the disease evolves over years into a more progressive course with or without intermixed relapses. This phase of disease is resistant to systemic immunotherapy, suggesting that mechanisms intrinsic to the CNS compartment underlie its development. The extent of ongoing remyelination is reported to be less apparent in the late chronic lesions which show the most extensive loss of myelin and OGCs. ${ }^{1}$ One speculates that the progressive disease phase reflects both continued injury and failure of repair processes.

The current study of Imitola et $\mathrm{al}^{2}$ in this issue of The American Journal of Pathology showing that susceptibility of pleuripotential neural stem cells to $T$ cell-mediated apoptosis involving a CD86-dependent mechanism can be viewed in the overall context of how the inflammatory process contributes to the remyelination or lack thereof that occurs in MS. An initial issue relates to the cellular source of the remyelination. In a number of rodent experimental demyelinating models involving use of toxins such as ethidium bromide or virus-induced immune-mediated demyelination such as Theiler's murine encephalomyelitis virus (TMEV), extensive remyelination is observed. ${ }^{3,4}$ This remyelination is attributed to recruitment of progenitor cells rather than to remyelination by previously myelinating OGCs. However, it is important to be cautious when extrapolating between species. For example, mature oligodendrocytes derived from the adult human CNS survive in long-term culture, while their rodent counterparts do not. ${ }^{5}$ Interestingly, studies of myelin basic protein (MBP) expression in mouse toxin models indicate that remyelination involves activation of a different set of myelin gene promoters versus initially myelinating cells. ${ }^{6}$ Successful remyelination by progenitor cells would be dependent not only on maturation of progenitor cells into mature myelinating cells but also on their capacity to migrate to the site of demyelination and to interact with a receptive axonal target.

The precise lineage followed as progenitor cells develop into mature OGCs continues to be defined. Myelinating cells may evolve from pleuripotential progenitor cells or progenitor cells that have already entered the neural or even glial lineage, as summarized in Figure 1. The pleuripotential progenitor cells are concentrated in the subependymal regions and are shown to be mobilized in response to acute inflammatory lesions in the experimental autoimmune encephalomyelitis (EAE) model. ${ }^{7}$ These are the cell populations studied in the report of Imitola et al. ${ }^{2}$ Progenitor cells expressing markers characteristic of immature OGCs have been identified and recovered from the non-inflamed adult human CNS pa-

Accepted for publication February 23, 2004.

Address reprint requests to Jack P. Antel, M.D., Montreal Neurological Institute, McGill University, 3801 University Street, Room 111, Montreal, Quebec, Canada C H3A 2B4. E-mail: jack.antel@mcgill.ca. 


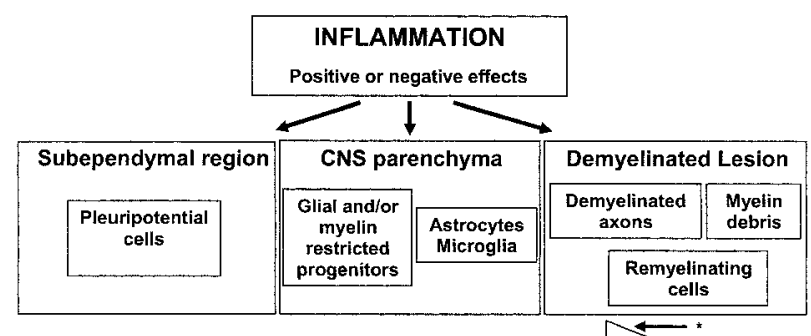

Progenitor cell migration and differentiation in the CNS

Figure 1. Effects of inflammation on progenitor cell-mediated remyelination in the central nervous system. Remyelination is dependent on migration of progenitor cells from either the subependymal region (pleuripotential cells) or from within the CNS parenchyma (glial/myelin-restricted progenitors) into the demyelinated lesions. The inflammatory response could have positive or negative effects on survival, migration, and differentiation of progenitor cells. * Dedifferentiation of mature OGCs may also result in cells expressing progenitor markers.

renchyma. $^{8,9}$ Recent data indicate that such progenitor cells can myelinate the CNS of shiverer mice, suggesting an intrinsic capability for myelination does exist. OGC progenitor cells are found in MS lesions, as defined by morphology and cell surface molecule expression criteria. ${ }^{10,11}$ The studies of Chang et $\mathrm{al}^{10}$ showing abundant numbers of progenitor cells even in chronic MS lesions, emphasize the issue that factors other than progenitor numbers alone contribute to the extent of remyelination that occurs.

Inflammatory mediators can impact either negatively or positively on progenitor cell-mediated remyelination via multiple mechanisms including direct effects on the cells, effects on the CNS environment (eg, astrocytes and microglia) that impact on progenitor cell migration and differentiation, and effects on the axons that are to be remyelinated (Figure 1). The most direct negative effect of the inflammatory response on progenitor cell-dependent remyelination would be immune-mediated injury to these cells. Selective injury of these cells could reflect the properties of either the immune mediators or the target cells. Within the inflammatory infiltrate of the active MS or EAE lesion are constituents of the adaptive immune system, either $\alpha \beta$ T cells or antibodies, which have the receptor diversity that would permit target selective recognition. There is at least one report of serum and/or cerebrospinal fluid (CSF) of MS patients having the capacity to injure progenitor cells due to presence of antibodies that recognize the NG2 epitope. ${ }^{12}$ Rosenbluth et $\mathrm{al}^{13}$ showed that implanting a hybridoma secreting IgM antibodies that recognize the sulfatide $\mathrm{O} 4$ expressed by oligodendrocyte lineage progenitor cells produced focal demyelination and that remyelination occurred only when the hybridoma degenerated. Conversely germ line anti-IgM antibodies can bind OGC progenitors and promote remyelination. $^{14}$

The inflammatory infiltrate also includes an array of cell bound (eg, death receptor ligands such as fas ligand and TRAIL) and soluble molecules (cytokines, proteases, excitotoxins) that can be produced by cells of the adaptive or innate immune system and can affect tissue injury in a non-antigen-restricted manner. ${ }^{15}$ Selective target susceptibility to such effectors would be dependent on spe- cific expression of requisite receptors for the effector molecules with induction of injury mediating signaling pathways. The array of molecules demonstrated to promote immune-neural interactions with consequences for non-antigen-restricted immune-mediated injury of neural cells continues to expand and now includes a range of adhesion molecules (eg, neural cell adhesion molecule (CD56) and intercellular adhesion molecules (CD54)). ${ }^{16,17}$ Giraudon et $\mathrm{al}^{18}$ recently showed that the apoptotic death of multipotential neural progenitor cells and immature OGCs induced by activated T cells could be inhibited by antibodies directed against SCD100/semaphorin 4D released by activated $T$ cells and reproduced with recombinant sCD100. This semaphorin was shown to collapse OGC process extension and to trigger apoptosis, most likely through receptors of the plexin family.

The report of Imitola et $\mathrm{al}^{2}$ defines a further mechanism whereby neural progenitor cells can become susceptible to injury or cell death because of expression of specific cell surface molecules that allow interaction with the immune system. Their in vitro studies using neural progenitor cell lines and their in vivo studies using the EAE model indicate that pluripotential neural stem cells up-regulate the expression of CD86 in response to inflammatory stimuli. CD86 is usually associated with antigen-presenting cells and is an essential co-stimulatory molecule regulating $T$ cell activation. CD80 and 86 expression by nestinpositive neural cells can be induced by the pro-inflammatory cytokines interferon $\gamma(\mathrm{IFN} \gamma)$ and tumor necrosis factor $\alpha(\mathrm{TNF} \alpha)$. Engagement of these molecules by $\mathrm{T}$ cells results in progenitor cell apoptosis. The constitutive and inducible expression of co-stimulatory molecules on progenitor cells would have the additional potential consequence of having these cells participate in antigen presentation to $T$ cells although one needs to document that these cells also express the appropriate $\mathrm{MHC}$ antigens. As mentioned, studies of the EAE model indicate that progenitor cells in the subventricular zone begin to be mobilized and to migrate to the lesion site following injury, presumably to participate in the repair process. The issue raised in the study of Imitola et $\mathrm{al}^{2}$ is whether injury of these neural cells could ultimately limit the extent of tissue repair that occurs in MS.

In regard to the impact of inflammation on generating a CNS environment that is or is not conducive for remyelination, most successful myelination related transplant experiments have involved the use of animals with genetic dysmyelination or toxin induced demyelination as recipients. Limited models exist in which transplants are performed in acute or chronic immune-mediated demyelinating disease. The myelin progenitor cells need to reach their targets to achieve effective myelination. Inflammation per se may be essential to removing injured tissue, especially myelin, which contains molecules that could inhibit cell migration or axonal regrowth. ${ }^{19} \mathrm{Re}$ cently, Aarum et $\mathrm{al}^{20}$ showed that migration and differentiation of neural precursor cells can be directed by soluble factors released from activated microglial cells that are concentrated at sites of injury in the CNS. Neural progenitor cells express receptors that permit response 
to chemoattractant molecules produced by inflammatory cells or by activated glial cells. Notably, the chemokine receptor CXCR2 controls positioning of OGC precursors in developing spinal cord by arresting their migration. ${ }^{21}$

OGC precursor cell migration is also directed by the secreted chemotropic guidance cue netrin-1. Netrin-1 is expressed at the ventral midline of the embryonic neural tube $^{22}$ and directs OGC precursors into dorsal and lateral regions of the developing spinal cord. ${ }^{23,24}$ Notably, netrin-1 induces the retraction of OGC precursor processes $^{23}$ an action proposed to underlie the function of netrin-1 as a chemorepellent. This may use mechanisms similar to that triggered by semaphorin CD $100 .{ }^{18}$ Interestingly, netrin expression has been detected in homogenates of spleen. ${ }^{22}$ Although the specific cell types involved have not been identified, this raises the possibility that netrins may regulate lymphocyte or macrophage motility. Mature myelinating OGCs in the adult CNS express netrin-1 and netrin-1 protein is normal component of periaxonal myelin, the interface between the axolemma and the inner face of the myelin sheath. ${ }^{25}$ Although it is not clear how netrins might be distributed in acute or chronic MS lesions, the presence of such guidance cues, either associated with myelin debris or expressed by invading immune cells, may influence the ability of OGC precursor cells to migrate into an MS lesion and remyelinate axons.

Successful progenitor cell-mediated remyelination would be dependent on the cells that reach the site of demyelination undergoing maturation into myelin-forming cells. Studies with systemic infusion of pleuripotential stem cells into EAE animals suggest that the enhanced remyelination that results reflects effects of soluble factors released by such cells on endogenous progenitor cells existent within the demyelinated regions. ${ }^{26}$ John et $\mathrm{al}^{27}$ reported that there was up-regulation of jagged expression in the MS lesions that lacked remyelination, a result attributed to increased production of transforming growth factor $\beta$ (TGF $\beta$ ) by astrocytes, providing an inhibitory signal for myelination. Remyelinated lesions had negligible levels of jagged expression. Notch 1 and Hes 5, the ligands for jagged are detected in immature OGCs.

In summary, the recent observation that remyelination is occurring in MS holds out the hope that enhanced understanding of the neurobiologic processes involved and how these are impacted by the process of inflammation, will result in development of therapeutic strategies that can be rapidly moved from the bench to the bedside.

\section{References}

1. Bruck W, Kuhlmann T, Stadelmann C: Remyelination in multiple sclerosis. J Neurol Sci 2003, 206:181-185

2. Imitola J, Comabella M, Chandraker AK, Dangond F, Seyegh MH, Snyder EY, Khoury SJ: Neural stem/progenitor cells express costimulatory molecules that are differentially regulated by inflammatory and apoptotic stimuli. Am J Pathol 2004, 164:1615-1625

3. Penderis J, Shields SA, Franklin RJM: Impaired remyelination and depletion of oligodendrocyte progenitors does not occur following repeated episodes of focal demyelination in the rat central nervous system. Brain 2003, 126:1382-1391.

4. Murray PD, McGavern DB, Sathornsumetee S, Rodriguez M: Spontaneous remyelination following extensive demyelination is associated with improved neurological function in a viral model of multiple sclerosis. Brain 2001, 124:1403-1416

5. Wosik K, Antel J, Kuhlmann T, Bruck W, Massie B, Nalbantoglu J: Oligodendrocyte injury in multiple sclerosis: a role for p53. J Neurochem 2003, 85:635-644

6. Farhadi HF, Lepage P, Forghani R, Friedman $\mathrm{HCH}$, Orfali W, Jasmin L, Miller W, Hudson TJ, Peterson AC: A combinatorial network of evolutionarily conserved myelin basic protein regulatory sequences confers distinct glial-specific phenotypes. J Neurosci 2003, 23: 10214-10223

7. Picard-Riera N, Decker L, Delarasse C, Goude K, Nait-Ournesmar B, Liblau R, Pham-Dinh D, Baron-Van Evercooren A: Experimental autoimmune encephalomyelitis mobilizes neural progenitors from the subventricular zone to undergo oligodendrogenesis in adult mice. Proc Natl Acad Sci USA 2002, 99:13211-13216

8. Chang A, Nishiyama A, Peterson J, Prineas J, Trapp BD: NG2positive oligodendrocyte progenitor cells in adult human brain and multiple sclerosis lesions. J Neurosci 2000, 20:6404-6412

9. Nunes MC, Roy NS, Keyoung HM, Goodman RR, McKhann G, Jiang L, Kang J, Nedergaard M, Goldman SA: Identification and isolation of multipotential neural progenitor cells from the subcortical white matter of the adult human brain. Nat Med 2003, 9:439-447

10. Chang A, Tourtellotte WW, Rudick R, Trapp BD: Premyelinating oligodendrocytes in chronic lesions of multiple sclerosis. N Engl $\mathrm{J}$ Med 2002, 346:165-173

11. Wolswijk G: Oligodendrocyte precursor cells in the demyelinated multiple sclerosis spinal cord. Brain 2002, 125:338-349

12. Niehaus A, Shi J, Grzenkowski M, Diers-Fenger M, Archelos J, Hartung HP, Toyka K, Bruck W, Trotter J: Patients with active relapsingremitting multiple sclerosis synthesize antibodies recognizing oligodendrocyte progenitor cell surface protein: implications for remyelination. Ann Neurol 2000, 48:362-371

13. Rosenbluth J, Schiff R, Liang WL, Dou W: Antibody-mediated CNS demyelination II. Focal spinal cord lesions induced by implantation of an IgM antisulfatide-secreting hybridoma. J Neurocytol 2003, 32: 265-276

14. Ciric B, Van K, V, Paz SM, Rodriguez M, Pease LR: Antibody-mediated remyelination operates through mechanism independent of immunomodulation. J Neuroimmunol 2004, 146:153-161

15. Antel JP, Arnold D: Multiple sclerosis. Neuroglia. Edited by Kettenmann H, Ransom B. Oxford University Press, 2004, in press

16. Antel JP, McCrea E, Ladiwala U, Qin YF, Becher B: Non-MHCrestricted cell-mediated lysis of human oligodendrocytes in vitro: relation with CD56 expression. J Immunol 1998, 160:1606-1611

17. Giuliani F, Goodyer CG, Antel JP, Yong VW: Vulnerability of human neurons to T cell-mediated cytotoxicity. J Immunol 2003, 171:368379

18. Giraudon $P$, Vincent $P$, Vuaillat $C$, Verlaeten $O$, Cartier L, MarieCardine A, Mutin M, Bensussan A, Belin MF, Boumsell L: Semaphorin CD100 from activated $T$ lymphocytes induces process extension collapse in oligodendrocytes and death of immature neural cells. J Immunol 2004, 172:1246-1255

19. Kalyvas A, David S: Cytosolic phospholipase A(2) plays a key role in the pathogenesis of multiple sclerosis-like disease. Neuron 2004, 41:323-335

20. Aarum J, Sandberg K, Haeberlein SL, Persson MA: Migration and differentiation of neural precursor cells can be directed by microglia. Proc Natl Acad Sci USA 2003, 100:15983-15988

21. Tsai HH, Frost E, To V, Robinson S, Ffrench-Constant C, Geertman R, Ransohoff RM, Miller RH: The chemokine receptor CXCR2 controls positioning of oligodendrocyte precursors in developing spinal cord by arresting their migration. Cell 2002, 110:373-383

22. Kennedy TE, Serafini T, de la Torre JR, Tessier-Lavigne M: Netrins are diffusible chemotropic factors for commissural axons in the embryonic spinal cord. Cell 1994, 78:425-435

23. Jarjour AA, Manitt C, Moore SW, Thompson KM, Yuh SJ, Kennedy TE: Netrin-1 is a chemorepellent for oligodendrocyte precursor cells in the embryonic spinal cord. J Neurosci 2003, 23:3735-3744 
24. Tsai HH, Tessier-Lavigne M, Miller RH: Netrin 1 mediates spinal cord oligodendrocyte precursor dispersal. Development 2003, 130:20952105

25. Manitt C, Colicos MA, Thompson KM, Rousselle E, Peterson AC, Kennedy TE: Widespread expression of netrin-1 by neurons and oligodendrocytes in the adult mammalian spinal cord. J Neurosci 2001, 21:3911-3922

26. Pluchino S, Quattrini A, Brambilla E, Gritti A, Salani G, Dina G, Galli
R, Del Carro U, Amadio S, Bergami A, Furlan R, Comi G, Vescovi AL, Martino G: Injection of adult neurospheres induces recovery in a chronic model of multiple sclerosis. Nature 2003, 422: 688-694

27. John GR, Shankar SL, Shafit-Zagardo B, Massimi A, Lee SC, Raine CS, Brosnan CF: Multiple sclerosis: re-expression of a developmental pathway that restricts oligodendrocyte maturation. Nat Med 2002, 8:1115-1121 